\title{
RADICAL PERINEAL PROSTATECTOMY (A CASE REPORT)
}

\author{
Hinev A. \\ Department of Surgery, Division of Urology, Varna Medical University, Varna, Bulgaria
}

Reviewed by: Assoc. Prof. K. Ivanov, MD, PhD

\begin{abstract}
INTRODUCTION: In view of the minimally invasive surgical techniques, gaining popularity in recent years, there has been a renewed interest by the urological community in radical perineal prostatectomy. MATERIAL AND METHODS: We present herein our first case with this technique. A 70-year-old male patient with biopsy proven prostate cancer underwent nerve sparing radical prostatectomy via the perineal approach. The surgical technique is described in details. RESULTS: The procedure was tolerated well by the patient, without any complications. He was discharged from the hospital on the 5th postoperative day and was closely followed-up after surgery. The patient restored his previous quality of life, remaining physically active, fully continent, and able to achieve sexual intercourse without the aid of additional pills or devices. His total PSA value fell to $0.01 \mathrm{ng} / \mathrm{ml}$ immediately after surgery, and maintained close to zero at the 3,6 and 9-month visits. CONCLUSION: Modern radical perineal prostatectomy may play an important role in the surgical treatment of localized prostate cancer, and it should be included in the training urological programs.
\end{abstract}

Keywords: prostate, prostate cancer, radical perineal prostatectomy

Despite the increased use of alternative treatment modalities (interstitial brachytherapy, cryotherapy, HIFU, etc.), radical prostatectomy continues to be the most common and perhaps the most definitive long term curative therapy for patients with localized, organ confined, prostatic carcinoma.

Radical prostatectomy can be accomplished through either a retropubic or perineal approach. The former surgical approach is currently preferred by most of the surgeons, as it allows simultaneous performance of pelvic (standard or extended) lymphadenectomy. Although initially applied for treatment of prostate cancer, the perineal technique lost its popularity throughout the years and became lost to a whole generation of trainees. In view of the recently developed minimally invasive surgical techniques, including the laparoscopic and robot assisted urological surgery, there has been a renewed interest by the urological community in radical perineal prostatectomy.

We herein present our first case of nerve sparing radical perineal prostatectomy, applied for localized prostate cancer.

\section{CASE REPORT}

A 70-year-old male patient (AMM, medical record 14570 /23.05.2007) presented in our clinic with mild lower urinary tract symptoms, which first appeared 1-2 years ago. In Feb-

Address for correspondence:

Alexander Hinev, Dept. of Surgery, Varna Medical University, 55

Marin Drinov Str., Varna 9002, Bulgaria

E-mail: ahinev@yahoo.com ruary 2007, the patient visited his local urologist, who performed DRE and US examination, and checked his total PSA value - $4.04 \mathrm{ng} / \mathrm{ml}$. Suspicious for prostate cancer, the urologist performed a fine needle aspiration prostate biopsy, which revealed the presence of a high differentiated prostate carcinoma (Biopsy No 232 / 26.02.2007). The patient was then referred to our clinic for radical surgical treatment.

The physical examination at admission showed a satisfactory patient common condition. The respiratory and cardio-vascular systems were without any pathological changes. The patient's blood pressure was $120 / 70 \mathrm{~mm} \mathrm{Hg}$, and his pulse frequency was 72 bpm. The liver and spleen were without any pathological changes. Succussio renalis was negative on both sides. The prostate on digital rectal examination was enlarged $(-4.0-4.5 \mathrm{~cm})$, with an irregular surface and firm consistence of both lobes.

Laboratory results: Hb 127 g/l; Hct 0.38; WBC 6.2 GI//; urea $6.8 \mathrm{mmol} / \mathrm{l}$; creatinine $89 \mu \mathrm{mol} / \mathrm{l}$; glucose $3.6 \mathrm{mmol} / \mathrm{l}$; AST 19 U/l; ALT 13 U/l; alkaline phosphatase 99 U/l; cholesterol $5.09 \mathrm{mmol} / \mathrm{l}$.

Urinalysis: protein (-); Microscopy: 5-6 RBC/HPF, 9-10 WBC/HPF. Urine culture: negative.

The ultrasound examination revealed normal structure of all abdominal organs. The prostate was enlarged (54/44/41 $\mathrm{mm})$, with multiple calcifications.

The preoperative pelvic CT (No 2497 / 19.03.2007) also confirmed an enlarged $(43 / 55 \mathrm{~mm})$ prostate, protruding into the bladder, with normal outer shape and heterogeneous structure. There were no pathologically enlarged pelvic lymph nodes. All bone structures were normal, without metastatic lesions. 
The chest Rö-graphy showed bilateral pneumofibrotic changes in the lungs, without any signs of pulmonary metastases.

The protocol concerning the management of the reported case was discussed and approved by the Advisory Board of Third Clinic of Surgery, "St. Marina" University Hospital, within which the surgery was undertaken. All procedures were carried out with the adequate understanding and written consent of the subject.

The patient underwent surgical exploration on May 28, 2007 (operative protocol No 405).

\section{SURGICAL TECHNIQUE}

Prior to prostatectomy, a standard mini-lymphadenectomy was first performed. It was implemented via a small $/ 5 \mathrm{~cm} /$ midline skin incision $2 \mathrm{~cm}$ above the pubic symphysis. Using long instruments with small working tips, wound retractors with long and narrow blades, and the so called "knot slider", which facilitated placement of the surgical knots deep into the wound, systematic bilateral dissection and removal of the pelvic lymph nodes from the external iliac and obturator regions was performed.

The patient was then placed on the operating table in an exaggerated lithotomy position, so that the peritoneum was brought into $45 \epsilon$ plane from horizontal. Patient draping included a rectal shield, which allowed permanent digital rectal control throughout the procedure. To increase the maneuverability of the prostate, a 22 Fr Foley catheter, stented by a rigid metal wire, was inserted into the bladder. To approach the prostate, we used the so called "suprasphincteric" route, developing a plane through the central tendon between the bulbocavernous muscle of the urethra and the sphincter ani externus muscle. A semicircular perineal skin incision was performed just above the anus, from one ischial tuberosity to the other. The underlying subcutaneous fatty tissue was divided by electrocautery. The muscular fibres of the subcutaneous portion of the external anal sphincter encountered in the midline were also sharply transected. The central tendon was then divided, exerting in the meantime a continuous caudal traction of the dorsal skin flap of the surgical wound. This maneuver enables the delineation of the muscular fibres of the rectum from those of the bulbospongiosus muscle. The rectourethralis muscle was incised transversely and the rectum was swept off the prostatic apex. The levator ani muscles on both sides were retracted laterally and the dorsal surface of the prostate, covered by the Denonvilliers' fascia, was liberated. A self-retaining wound retractor was now installed to replace the assistant's hands.

An inverted T-shaped incision on the Denonvilliers' fascia was made, taking care not to damage the neurovascular bundles, passing along the posterolateral aspects of the prostate. The neurovascular bundles on both sides were dissected cranially towards the apex and a little bit past the urethroprostatic junction, and caudally well off the tips of the seminal vesicles. Each vas deferens was divided and ligated, as far from the prostate, as possible. The seminal vesicles were then consecutively identified and dissected free on either side. By pulling them in a contralateral and cranial direction, the prostatic vascular pedicles became easily evident. They were transected between right-angle clamps. The dissection continued on the bladder neck, behind the prostate, starting from the midline and following laterally on both sides.

When this dissection was completed, the dorsal circumference of the urethra was mobilized and incised. The Foley catheter was exteriorized through the urethral incision and pulled upwards to improve visualization of the urethroprostatic junction. Another Foley catheter was then inserted into the bladder and used to push the prostate downwards. The urethral stamp was then transected completely. The puboprostatic ligaments were identified and divided. The dorsal vein complex was swept off the prostate, and, by pushing the prostate downwards, its anterior surface was completely dissected. The prostatovesical junction was identified and dissected. The bladder neck was incised and the tip of the Foley catheter was exteriorized through the incision. Then, the remaining bladder neck circumference was incised, and the whole specimen was removed.

Using 3-0 polyglactin sutures, the bladder neck was reconstructed in a "tennis racket" fashion. To avoid strictures at the anastomotic site, the bladder mucosa at the remaining opening was everted and fixed by four 5-0 absorbable sutures. The urethrovesical anastomosis was completed under direct vision. Initially, four separate 3-0 polyglactin sutures were placed and tied in sequence at the 10,11,1, and $2 o^{\prime}$ clock positions. Then, a silicone Foley catheter $22 \mathrm{Fr}$ was inserted transurethrally and positioned intravesically by inflating the balloon with $15 \mathrm{ml}$ of saline. The dorsal circumference of the anastomosis was completed by placing additional four sutures at the 4, 8, 5 and 7 o'clock positions. The water-tightness of the anastomosis was controlled by filling the bladder with $300 \mathrm{ml}$ of saline. Two rubber drains were left in place on both sides of the urethrovesical anastomosis and the pelvic floor was reconstructed by readapting the levator ani muscles in the midline. The subcutaneous fatty tissue and the skin were sutured by interrupted 3-0 polyglactin sutures.

The final histological examination (No 5471-5483/ 7.06.2007) confirmed the presence of adenocarcinoma (Gleason score 6/3+3/), localized in the prostate. All lymph nodes and surgical margins sent for histological examination were cancer negative. The pathological stage was assessed as T2c N0 M0.

\section{RESULTS}

The patient tolerated the procedure well. He was allowed to receive liquids and ambulate on the first day after surgery. The regular diet was initiated on the second day. The drains were removed 2 days after surgery. The patient was discharged from the hospital with a primarily healed surgical wound on the 5th postoperative day (on June 2, 2007).

The patient was closely followed-up after surgery. The Foley catheter was removed 1 week after the procedure. The patient restored normal voiding, with a broad urinary stream. Mild stress incontinence was recorded in the first 
few days after removal of the catheter. Therefore, pelvic floor training was initiated immediately, with rapid and excellent results.

On the second postoperative visit (3 weeks after surgery) the patient reported to be completely continent, both at day and at night time. He also reported to have occasional spontaneous erections in the moming, although he still avoided sexual intercourse. On the 3-month postoperative visit the patient reported that he achieved his first successful sexual intercourse after surgery, without the aid of any additional pills or devices.

The measured check-up level of patient's total PSA was $0.01 \mathrm{ng} / \mathrm{ml}$ immediately after surgery, and it maintained close to zero at the 3, 6 and 9-month visits.

\section{DISCUSSION}

The perineal approach for surgical cure of prostate cancer was first introduced by Young in the early 1904 and published in 1905 (12). It dominated in the literature until 1945, when Millen described the retropubic approach to perform prostatectomy (4). Applied initially for BPH, the retropubic approach was further developed to treat prostate cancer. However, for many years radical prostatectomy was not commonly performed by either one of the two approaches proposed, because of the high complication rate associated with the procedure, such as: impotence, incontinence, and bleeding. A real break-through in the surgical management of prostate cancer occurred in the early 1980s, when Walsh et al. described in details the anatomical nerve sparing retropubic technique, which turned to be the gold standard operation for localized prostate cancer (10).

In the age of minimally invasive surgery there has been renewed interest in the perineal approach for the surgical treatment of organ-confined prostate cancer (2). This operation offers to the surgeon the benefits of lower blood loss, decreased postoperative pain, shorter hospital stay and more rapid recovery and return to work, as compared with the radical retropubic prostatectomy $(1,2,8)$. The procedure is much shorter and less expensive than the laparoscopic and robot assisted radical prostatectomy, meanwhile providing similar functional and oncological outcomes (7).

The modifications of the surgical technique, added by Weldon and Tavel in 1988, introduced the modern anatomical principles, and mainly the nerve sparing approach that made the postoperative results similar to those achieved by the retropubic technique (11).

A major disadvantage of RPP is that lymphadenectomy cannot be performed through the same incision. However, the wide spread use of PSA led to an early detection of the disease by multiple prostate biopsies and dramatically decreased the incidence of positive lymph nodes found at surgery (6). Besides, lymphadenectomy can be now performed by less invasive surgical techniques: via a minimal suprapubic skin incision (the so called mini-lymphadenectomy), by the laparoscopic technique (9), or by the recently developed innovations in the technique of perineal prostatectomy - via the same perineal incision $(3,5)$.
In conclusion, the modern radical perineal prostatectomy may play an important role in the surgical treatment of localized prostate cancer. It proved to be technically feasible, safe and effective, with incontinence and erectile dysfunction rates similar to those of radical retropubic prostatectomy. It is less invasive and leads to more rapid resumption of physical activity and return to work than the retropubic approach. Therefore, it should be an option in the armamentarium of contemporary surgical techniques used to eradicate prostate cancer, and for this reason it should be included in the training urological programs.

\section{REFERENCES}

1. Harris, M. The anatomic radical perineal prostatectomy: an outcomes-based evolution. -Eur. Urol., 52(1), 2007, 81-88.

2. Janoff, D., R. Parra. Contemporary appraisal of radical perineal prostatectomy. - J. Urol., 173(6), 2005, 1863-1870.

3. Keller, H., J. Lehmann, J. Beier. Radical perineal prostatectomy and simultaneous extended pelvic lymph node dissection via the same incision. - Eur. Urol., 52(2), 2007, 384-388.

4. Millen, T. Retropubic prostatectomy, a new extravesical technique. - Lancet, 2, 1945, 693.

5. Saito, S., G. Murakami. Radical perineal prostatectomy: a novel approach for lymphadenectomy from perineal incision. - J. Urol., 170(4 Pt 1), 2003, 1298-1300.

6. Salomon, L., A. Hoznek, M. Lefrere-Belda, J. Bellot, D. Chopin, C. Abbou. Nondissection of pelvic lymph nodes does not influence the results of perineal radical prostatectomy in selected patients. - Eur. Urol., 37(3), 2000, 297-300.

7. Salomon, L., O. Levrel, A. de la Taille, A. Anastasiadis, F. Saint, S. Zaki et al. Radical prostatectomy by the retropubic, perineal and laparoscopic approach: 12 years of experience in one center. - Eur. Urol., 42(2), 2002, 104-110.

8. Sullivan, L., M. Weir, J. Kinahan, D. Taylor. A comparison of the relative merits of radical perineal and radical retropubic prostatectomy. - BJU Int., 85(1), 2000, 95-100.

9. Thomas, R., R. Steele, R. Smith, W. Brannan. One-stage laparoscopic pelvic lymphadenectomy and radical perineal prostatectomy. - J. Urol., 152(4), 1994, 1174-1177.

10. Walsh, P., H. Lepor, J. Eggleston. Radical prostatectomy with preservation of sexual function: anatomical and pathological considerations. - Prostate, 4(5), 1983, 473-485.

11. Weldon, V., F. Tavel. Potency-sparing radical perineal prostatectomy: anatomy, surgical technique and initial results. - J. Urol., 140(3), 1988, 559-562.

12. Young, H. The early diagnosis and radical cure of carcinoma of the prostate. Being a study of 40 cases and presentation of a radical operation which was carried out in four cases. - Johns Hopkins Hosp. Bull., 16, 1905, 315 . 